\title{
Chitosan promotes immune responses, ameliorates glutamic oxaloacetic transaminase and glutamic pyruvic transaminase, but enhances lactate dehydrogenase levels in normal mice in vivo
}

\author{
MING-YANG YEH ${ }^{1 *}$, YUNG-LUEN SHIH ${ }^{2-4 *}$, HSUEH-YU CHUNG ${ }^{5}$, JASON CHOU $^{6}, \mathrm{HSU}^{2} \mathrm{FENG} \mathrm{LU}^{7}$, \\ CHIA-HUI LIU ${ }^{8}$, JIA-YOU LIU ${ }^{7}$, WEN-WEN HUANG ${ }^{9}$, SHU-FEN PENG ${ }^{9}$, \\ LUNG-YUAN WU ${ }^{10}$ and JING-GUNG CHUNG ${ }^{9,11}$
}

\author{
${ }^{1}$ Office of Director, Cheng Hsin General Hospital; ${ }^{2}$ Department of School of Medicine, Fu-Jen Catholic University; \\ ${ }^{3}$ Department of Pathology and Laboratory Medicine, Shin Kong Wu Ho-Su Memorial Hospital; \\ ${ }^{4}$ School of Medical Laboratory Science and Biotechnology, Taipei Medical University, Taipei; ${ }^{5}$ Jen-Teh Junior College of \\ Medicine, Nursing and Management, Miaoli; Departments of ${ }^{6}$ Anatomical and ${ }^{7}$ Clinical Pathology, \\ Cheng Hsin General Hospital, Taipei; ${ }^{8}$ The Center of General Education, Chia-Nan University of Pharmacy and Science, \\ Tainan; ${ }^{9}$ Department of Biological Science and Technology, China Medical University, Taichung; \\ ${ }^{10}$ The School of Chinese Medicine for Post Baccalaureate, I-Shou University, Kaohsiung; \\ ${ }^{11}$ Department of Biotechnology, Asia University, Taichung, Taiwan, R.O.C.
}

Received February 2, 2015; Accepted January 15, 2016

DOI: $10.3892 /$ etm.2016.3057

\begin{abstract}
Chitosan, a naturally derived polymer, has been shown to possess antimicrobial and anti-inflammatory properties; however, little is known about the effect of chitosan on the immune responses and glutamic oxaloacetic transaminase (GOT), glutamic pyruvic transaminase (GPT) and lactate dehydrogenase (LDH) activities in normal mice. The aim of the present study was to investigate whether chitosan has an effect on the immune responses and GOT, GPT and LDH activities in mice in vivo. BALB/c mice were divided into four groups. The negative control group was treated with a normal diet; the positive control group was treated with a normal diet plus orally administered acetic acid and two treatment groups were treated with a normal diet plus orally administered chitosan in acetic acid at doses of 5 and $20 \mathrm{mg} / \mathrm{kg}$, respectively, every other day for 24 days.
\end{abstract}

Correspondence to: Professor Jing-Gung Chung, Department of Biological Science and Technology, China Medical University, 91 Hsueh-Shih Road, Taichung 40402, Taiwan, R.O.C.

E-mail: jgchung@mail.cmu.edu.tw

Dr Lung-Yuan Wu, The School of Chinese Medicine for Post-Baccalaureate, I-Shou University (Yanchao Campus), 8 Yida Road, Jiaosu, Yanchao, Kaohsiung 82445, Taiwan, R.O.C.

E-mail: dr.wuly@gmail.com

* Contributed equally

Key words: chitosan, immune responses, glutamic oxaloacetic transaminase, glutamic pyruvic transaminase, lactate dehydrogenase
Mice were weighed during the treatment, and following the treatment, blood was collected, and liver and spleen samples were isolated and weighted. The blood samples were used for measurement of white blood cell markers, and the spleen samples were used for analysis of phagocytosis, natural killer (NK) cell activity and cell proliferation using flow cytometry. The results indicated that chitosan did not markedly affect the body, liver and spleen weights at either dose. Chitosan increased the percentages of CD3 (T-cell marker), CD19 (B-cell marker), CD11b (monocytes) and Mac-3 (macrophages) when compared with the control group. However, chitosan did not affect the phagocytic activity of macrophages in peripheral blood mononuclear cells, although it decreased it in the peritoneal cavity. Treatment with $20 \mathrm{mg} / \mathrm{kg}$ chitosan led to a reduction in the cytotoxic activity of NK cells at an effector to target ratio of 25:1. Chitosan did not significantly promote B-cell proliferation in lipopolysaccharide-pretreated cells, but significantly decreased T-cell proliferation in concanavalin A-pretreated cells, and decreased the activity of GOT and GPT compared with that in the acetic acid-treated group,. In addition, it significantly increased LDH activity, to a level similar to that in normal mice, indicating that chitosan can protect against liver injury.

\section{Introduction}

The emergence and growth of tumors are known to be associated with tumor immunosurveillance and antitumor immune responses (1). However, one of the drawbacks of many therapeutic technologies for cancer patients is the inadvertent induction of host immune responses (2). Thus, previous studies have focused on immune-mediated protection against cancer in immunocompromised patients with cancer and mouse 
models (3). Treatments for human cancers remain a therapeutic challenge, and the identification and development of novel agents to induce immune function is necessary.

Chitosan, a linear heteropolysaccharide composed of $\beta$ - $(1,4)$-linked D-glucosamine $(\mathrm{GlcN})$ and $\beta$ - $(1,4)$-linked D-N-acetylglucosamine (GlcNAc), can be derived from chitin (4), which is a naturally occurring polysaccharide composed of GlcNAc units (5). Chitosan can be used as a biomaterial for tissue regeneration, and has antibacterial, anti-inflammatory and drug delivery functions (6). Numerous studies have demonstrated that chitosan may inhibit the growth of microbial organisms, such as Porphyromonas gingivalis (7), Actinobacillus actinomycetemcomitans, Streptococcus mutans $(8,9)$, Pseudomonas aeroginosa, Staphylococcus aureus (10) and Aggregatibacter actinomycetemcomitans (11).

In human astrocytoma cells, the secretion and expression of the pro-inflammatory cytokines tumor necrosis factor (TNF) $-\alpha$ and interleukin (IL) 6 has been shown to be markedly inhibited following pretreatment with water-soluble chitosan (9). It has also been reported that chitosan-induced macrophages exhibit markedly downregulated expression of pro-inflammatory markers, such as cluster of differentiation CD86 and major histocompatibility complex II (MHCII), and decrease the expression of pro-inflammatory cytokines, specifically TNF- $\alpha$; however, the anti-inflammatory markers IL-10 and TGF- $\beta 1$ were found to be increased $(12,13)$.

Despite the reports of several studies that chitosan has an anti-inflammatory effect in vitro, knowledge concerning the effect of chitosan on the immune responses of normal mice is lacking. In the present study, the promoted immune responses in BALB/c mice were evaluated in vivo. Furthermore, the levels of certain enzymes, including glutamic oxaloacetic transaminase (GOT), glutamic pyruvic transaminase (GPT) and lactate dehydrogenase (LDH), were analyzed in BALB/c mice following oral treatment with chitosan. The expression levels of the white blood cell markers CD3, CD11b, CD19 and Mac-3 were also investigated.

\section{Materials and methods}

Materials and reagents. Acetic acid was obtained from Sigma-Aldrich (St. Louis, MO, USA). RPMI-1640 medium, fetal bovine serum, L-glutamine and penicillin-streptomycin were purchased from Gibco (Thermo Fisher Scientific, Inc., Waltham, MA, USA). Tissue culture plastic wares and Tissue culture plastic wares and phycoerythrin (PE)-conjugated anti-mouse-CD3 (cat. no. 553062), PE-conjugated anti-mouse-CD19 (cat. no. 553786), FITC-conjugated anti-mouse-CD11b (cat. no. 553310) and FITC-conjugated anti-mouse-Mac-3 (cat. no. 553322) were purchased from BD Pharmigen (San Diego, CA, USA).

Preparation of chitosan. Chitosan powder with a molecular weight of $\sim 86,000 \mathrm{kDa}$ (Koyo Chemical Co., Ltd., Sakaiminato, Japan) was obtained from the National Taiwan University College of Medicine Animal Medicine Center (Taipei, Taiwan). The doses of 5 and $20 \mathrm{mg} / \mathrm{kg}$ were separately suspended in $0.2 \mathrm{ml}$ acetic acid for $1 \mathrm{~h}$ at room temperature prior to use (14).
Male BALB/c mice and chitosan treatment. Forty male BALB/c mice aged 4 weeks and weighing 22-25 g, were obtained from the National Laboratory Animal Center (Taipei, Taiwan). All mice were maintained at $25^{\circ} \mathrm{C}$ on $12 \mathrm{~h}$ light/dark cycles in the animal center of China Medical University (Taichung, Taiwan). All animal experiments were reviewed and approved by the Institutional Animal Care and Use Committee of China Medical University (approval ID, 103-215-B). All animal care was in accordance with the institutional animal ethical guidelines of the China Medical University (15). The 40 mice were randomly divided into the following four groups (10 mice per group): Negative control group, comprising untreated mice; positive control group, treated with acetic acid; $5 \mathrm{mg} / \mathrm{kg}$ group, treated with $5 \mathrm{mg} / \mathrm{kg}$ chitosan in acetic acid, and $20 \mathrm{mg} / \mathrm{kg}$ group, treated with $20 \mathrm{mg} / \mathrm{kg}$ chitosan in acetic acid. Mice in all four groups were fed a normal diet. Chitosan in acetic acid was administered by gavage every 2 days for a total of 24 days (12 times), during which the body weight was recorded. Upon termination of the treatment, mice from each group were weighed and sacrificed with $\mathrm{CO}_{2}$, as previously described (15).

Immunofluorescence staining for surface markers. Upon termination of the treatment, all mice were individually weighed and blood samples, as well as the liver and spleen of the mice were individually collected. The collected spleens were used for the isolation of splenocytes and measurement of natural killer (NK) cell activity, as previously described (15). A blood sample of $1 \mathrm{ml}$ from each mouse was lysed to destroy the red blood cells using 1X BD Pharm Lyse ${ }^{\mathrm{TM}}$ lysing buffer (BD Biosciences, Franklin Lakes, NJ, USA) according to the manufacturer's protocol, and leukocytes were collected as previously described (15). Phycoerythrin (PE)-labeled anti-mouse CD3, PE-labeled anti-mouse CD19, fluorescein isothiocyanate (FITC)-labeled anti-mouse CD11b and FITC-labeled anti-mouse Mac-3 antibodies (all dilution 1:40) were used to stain the isolated leukocytes for $30 \mathrm{~min}$, and then all samples were washed with phosphate-buffered saline (PBS). After this, all samples were analyzed using flow cytometry (BD FACSCalibur; BD Biosciences) to measure the percentages of white blood cell markers, as previously described (15).

Measurements of the phagocytic activity of macrophages. Macrophages were isolated from the peripheral blood mononuclear cells (PBMCs) and peritoneum of each mouse as previously described (15) and were placed in plates containing $50 \mu$ larget $E$. coli-FITC according to PHAGOTEST ${ }^{\circledR}$ kit manufacturer's instructions (ORPEGEN Pharma $\mathrm{GmbH}$, Heidelberg, Germany). All samples were individually mixed, then examined for phagocytosis using flow cytometery. Quantification of phagocytosis was performed using CellQuest software (version 5.1; BD Biosciences) as previously described (15).

Measurements of NK cell cytotoxic activity. Splenocytes were isolated from each spleen as previously described (15) and were placed in 96 -well plates $\left(1 \times 10^{5}\right.$ cells/well $)$ with $1 \mathrm{ml}$ RPMI-1640 medium. Target YAC- 1 cells $\left(2.5 \times 10^{7}\right.$ cells; Food Industry Research and Development Institute, Hsinchu, Taiwan) and PKH-67/Diluent C buffer (Sigma-Aldrich) were individually added to the cell-containing wells, according 

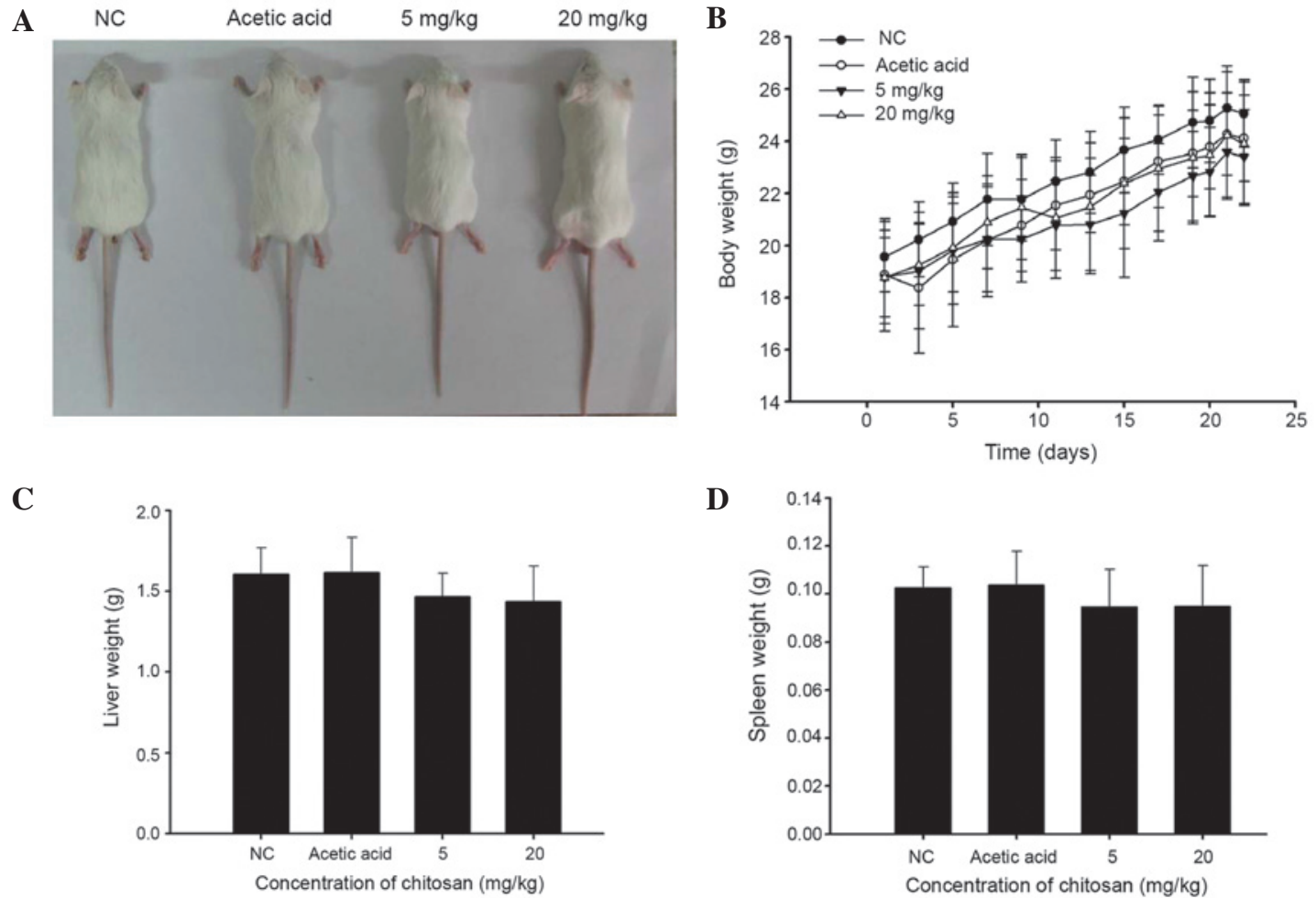

Figure 1. Effects of chitosan on the appearance, and body, liver and spleen weights of normal BALB/c mice. The NC group was fed a normal diet; the acetic acid group was fed a normal diet and acetic acid; the $5 \mathrm{mg} / \mathrm{kg}$ group was fed a normal diet and $5 \mathrm{mg} / \mathrm{kg}$ chitosan in acetic acid, and the $10 \mathrm{mg} / \mathrm{kg}$ group was fed a normal diet and $20 \mathrm{mg} / \mathrm{kg}$ chitosan in acetic acid. All animals were treated for gavage every 2 days for a total of 24 days (12 times). (A) Animal appearance and (B) body, (C) liver and (D) spleen weights of the mice in the four groups. The total body weights were measured every 2 days. NC, negative control.

to the manufacturer's protocol. The samples were mixed thoroughly for $2 \mathrm{~min}$ at $25^{\circ} \mathrm{C}$ and $2 \mathrm{ml} \mathrm{PBS}$ was added to each well for $1 \mathrm{~min}$ together with $4 \mathrm{ml}$ medium. The mix was then incubated for $10 \mathrm{~min}$. Following incubation, all samples were centrifuged for $2 \mathrm{~min}$ at $290 \mathrm{x} \mathrm{g} \mathrm{rpm}\left(25^{\circ} \mathrm{C}\right)$. NK cell cytotoxic activity was measured using flow cytometry as previously described (15).

Measurements of $T$ - and B-cell proliferation. Isolated splenocytes $\left(1 \times 10^{5}\right.$ cells/well) from each mouse were placed in 96-well plates containing $100 \mu \mathrm{l}$ RPMI-1640 medium. Following stimulation by incubation with concanavalin A (Con A; $0.5 \mu \mathrm{g} / \mathrm{ml}$; Sigma-Aldrich) for 3 days, T-cell proliferation was measured. In addition, B-cell proliferation was measured following stimulation with lipopolysaccharide (LPS, $1 \mu \mathrm{g} / \mathrm{ml}$; Sigma-Aldrich) for 5 days. Cell proliferation was measured using CellTiter 96 AQueous One Solution Cell Proliferation Assay kit (Promega Corporation, Madison, WI, USA) as previously described (15).

Measurement of blood levels of GOT, GPT and LDH in $B A L B / c$ mice following exposure to chitosan. Following treatment, blood samples were collected from all mice in order to measure the levels of GOT, GTP and LDH using quantitative kits. The kits were liquiUV (aspartate aminotransferase) for GOT, liquiUV (alanine aminotransferase) for GPT and liquiUV (lactate dehydrogenase) for LDH, respectively, which were purchased from HUMAN Gesellschaft für Biochemica und Diagnostica mbH (Wiesbaden, Germany), and were used as previously described $(16,17)$.
Statistical analysis. The data from three independent experiments were expressed as the mean \pm standard error. Statistical comparison between the chitosan and control groups was performed using the Student's t-test. $\mathrm{P}<0.05$ was considered to indicate a statistically significant difference.

\section{Results}

Chitosan affects the body, liver and spleen weights of normal $B A L B / c$ mice. Representative images of the mice in the four groups, and animal body, liver and spleen weights are presented in Fig. 1. These results indicate that chitosan did not significantly affect the appearance of the animals (Fig. 1A) or the body (Fig. 1B), liver (Fig. 1C) or spleen (Fig. 1D) weights when compared with those of the vehicle-treated group.

Chitosan affects cell markers of white blood cells in normal $B A L B / c$ mice. Blood samples were collected from each mouse and the levels of CD3, CD19, CD11b and Mac-3 cell markers were measured. The results presented in Fig. 2 indicate that chitosan promoted CD3 (Fig. 2A), CD19 (Fig. 2B), CD11b (Fig. 2C) and Mac-3 (Fig. 2D) expression at both doses, when compared with the acetic acid-treated group.

Chitosan affects the phagocytic activity of macrophages from the PBMCs and peritoneal cavity of normal BALB/c mice. Following treatment, cells were isolated from the PBMCs and peritoneal cavity of each animal, in order to measure the percentage of phagocytosis and results are shown in Fig. 3A and B, respectively. Neither of the two doses of chitosan (5 and 
A

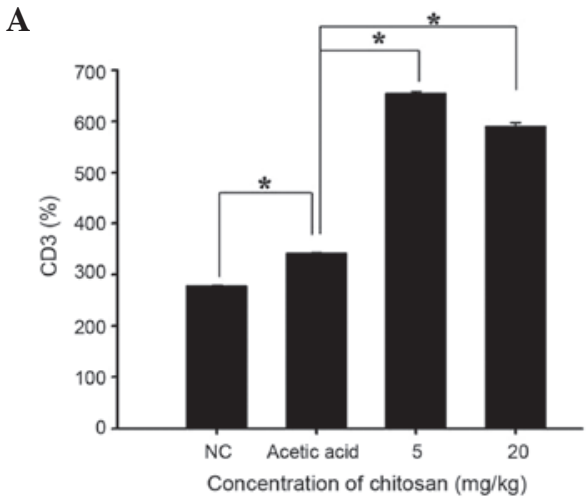

C

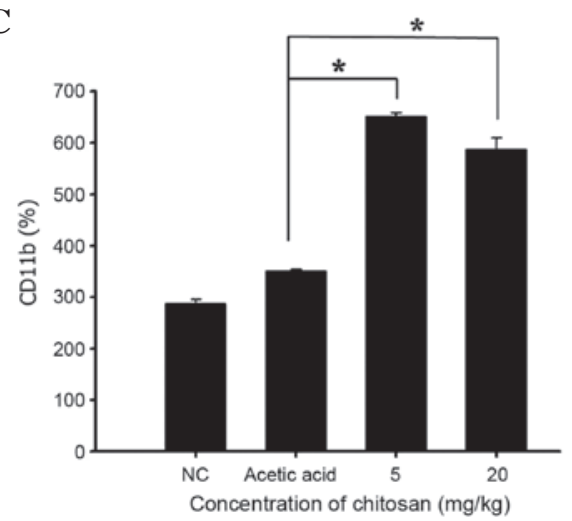

B

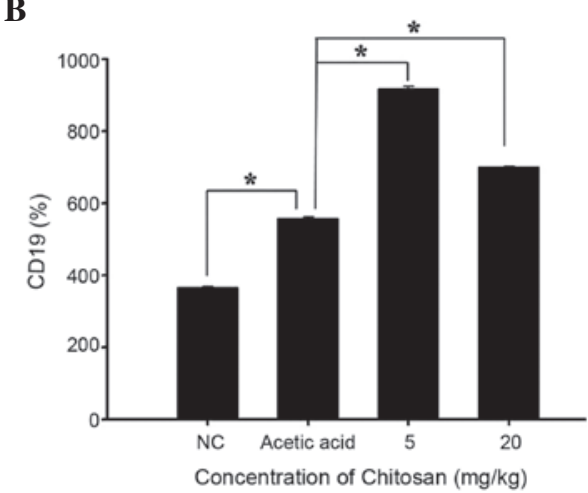

D

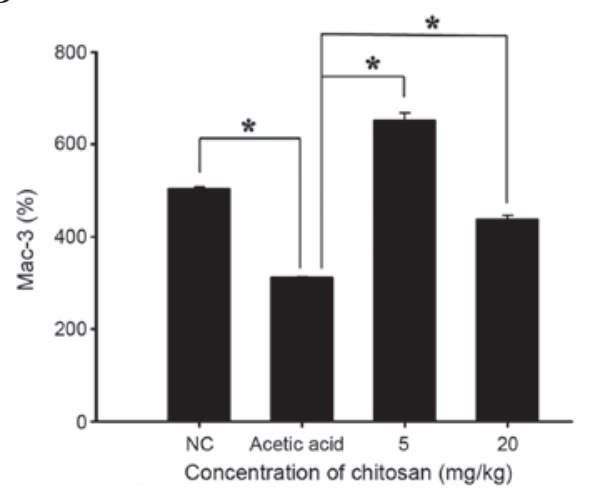

Figure 2. Chitosan affected the levels of white blood cell markers in normal BALB/c mice. Blood samples were collected from all mice and analyzed for (A) CD3, (B) CD19, (C) CD11b and (D) Mac-3 cell markers using flow cytometry. Data are expressed as the mean \pm standard error of three experiments (n=10). ${ }^{*} \mathrm{P}<0.05$, for the comparisons indicated. $\mathrm{CD}$, cluster of differentiation; NC, negative control.

A

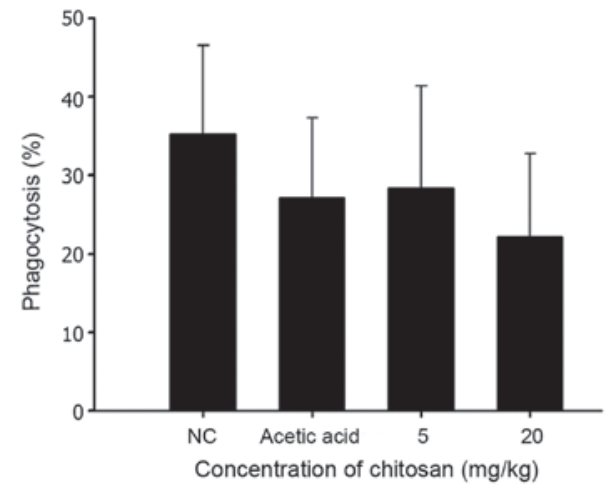

B

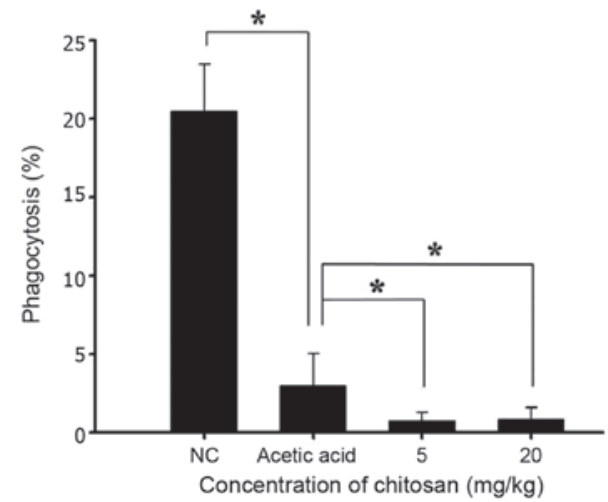

Figure 3. Chitosan decreased the phagocytic activity of macrophages from the PBMCs and peritoneal cavity of normal BALB/c mice. Macrophages were isolated from the (A) PBMCs and (B) peritoneum of each mouse, and macrophage phagocytosis was measured using flow cytometery and quantified using CellQuest software. ${ }^{*} \mathrm{P}<0.05$, for the comparisons indicated. $\mathrm{NC}$, negative control.

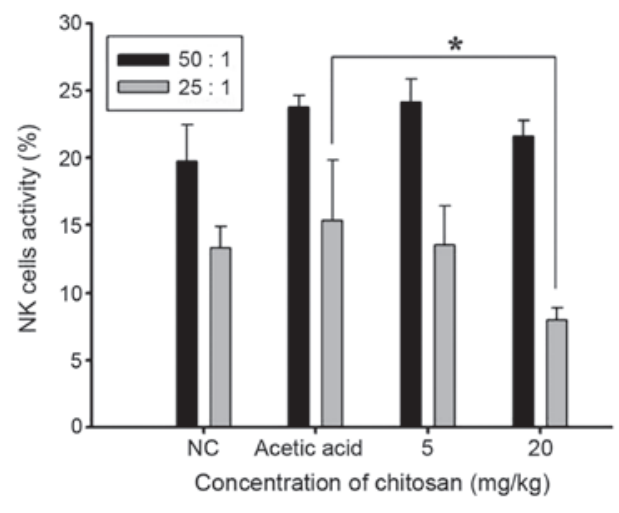

Figure 4. Chitosan affected the cytotoxic activity of NK cells in normal $\mathrm{BALB} / \mathrm{c}$ mice. YAC-1 target cells were incubated with isolated splenocytes from the mice and NK cell cytotoxic activity was measured using flow cytometry. ${ }^{*} \mathrm{P}<0.05$, for the comparisons indicated. NK, natural killer; $\mathrm{NC}$, negative control.

$20 \mathrm{mg} / \mathrm{kg}$ ) was found to significantly affect phagocytosis by macrophages from PMBCs (Fig. 3A); however, both doses were found to decrease phagocytosis by macrophages from the peritoneal cavity (Fig. 3B).

Chitosan affects the cytotoxic activity of $\mathrm{NK}$ cells from normal BALB/c mice. YAC-1 cells were used as targets for isolated splenocytes and were examined using flow cytometry. The results (Fig. 4) indicated that chitosan did not significantly affect the cytotoxic activity of NK cells at an effector to target ratio of 50:1; however, at the dose of $20 \mathrm{mg} / \mathrm{kg}$ chitosan and an 
A

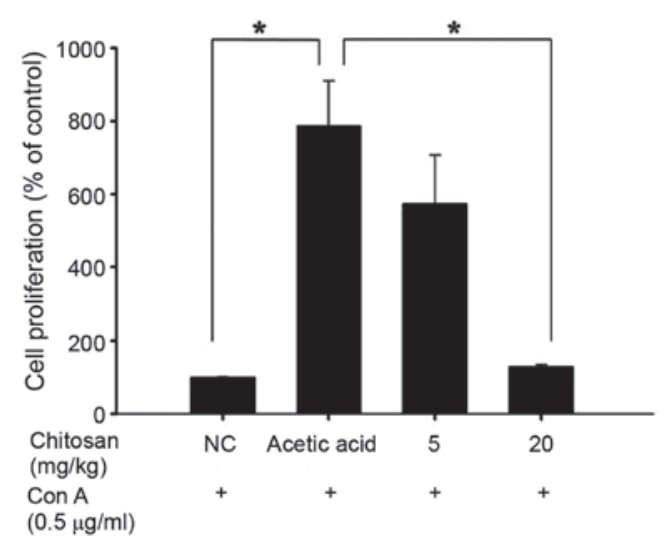

B

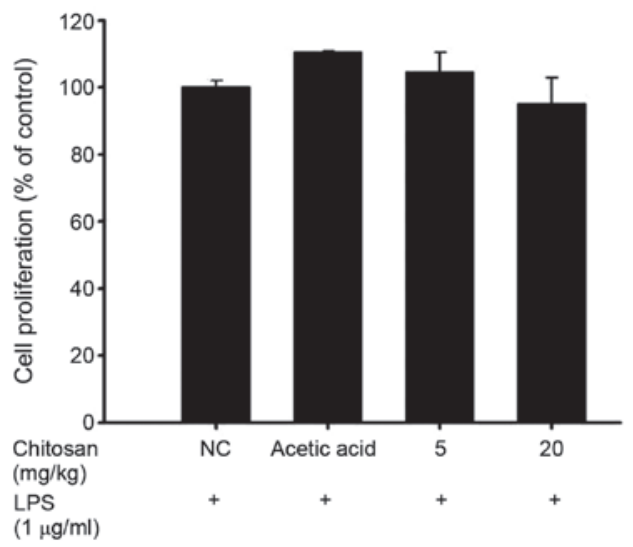

Figure 5. Chitosan affected T and B cell proliferation in normal BALB/c mice. Isolated T and B cells were pretreated with Con A and LPS for the induction of (A) T-cell and (B) B-cell proliferation, respectively, and were then analyzed using flow cytometry. ${ }^{*} \mathrm{P}<0.05$, for the comparisons indicated. Con A, concanavalin A; LPS, lipopolysaccharide; NC, negative control.

A

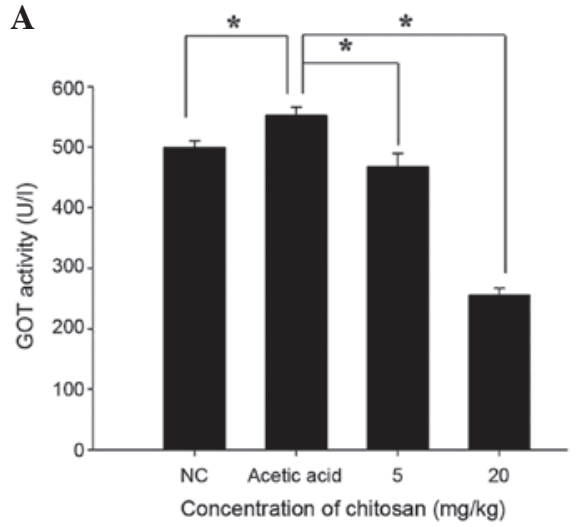

B

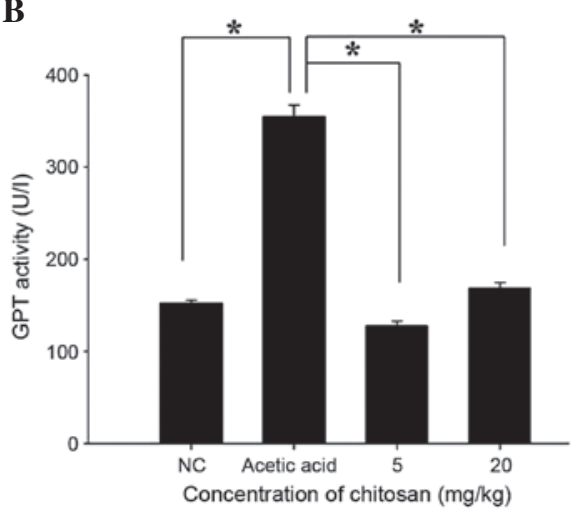

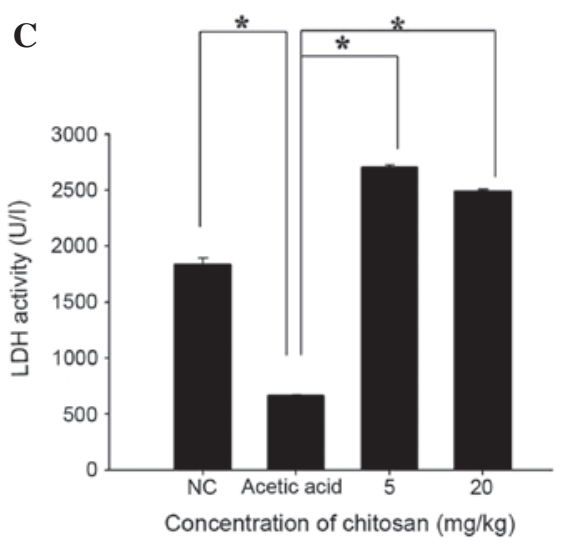

Figure 6. Measurement of GOT, GPT, LDH activity in the blood of BALB/c mice following exposure to chitosan. Following treatment, blood samples were collected from each mouse and the activities of (A) GOT, (B) GTP and (C) LDH were measured using commercially available kits. * $<<0.05$, for the comparisons indicated. GOT, glutamic oxaloacetic transaminase; GPT, glutamic pyruvic transaminase; LDH, lactate dehydrogenase; NC, negative control.

effector to target ratio of 25:1 led to a significant reduction in the cytotoxic activity of the NK cells when compared with that in the acetic acid-treated group $(\mathrm{P}<0.05$; Fig. 4).

Chitosan affects $T$ - and $B$-cell proliferation in normal $B A L B / C$ mice. Isolated splenocytes were assayed for T- and B-cell proliferation using flow cytometry and the results are presented in Fig. 5. The two chitosan doses (5 and $20 \mathrm{mg} / \mathrm{kg}$ ) notably decreased $\mathrm{T}$ cell proliferation when compared with the acetic acid-treated group (Fig. 5A), but did not significantly affect B-cell proliferation (Fig. 5B).

Chitosan affects the activity of blood enzymes GOT, GPT and $L D H$ in $B A L B / c$ mice. Following treatment, the mice were sacrificed and blood samples were collected from each mouse in order to measure the activity of GOT, GTP and LDH (Fig. 6). Chitosan significantly decreased GOT and GPT activity when compared with that in the acetic acid-treated group $(\mathrm{P}<0.05$; Fig 6A and B). However, GPT activity in the $20 \mathrm{mg} / \mathrm{kg}$ chitosan group was slightly higher than that in normal mice. Furthermore, chitosan significantly increased LDH activity when compared with that in the acetic acid-treated group $(\mathrm{P}<0.05)$.

\section{Discussion}

Although numerous studies have shown that chitosan is able to inhibit the growth of microbial organisms (7-11), it has also been shown to cause significant downregulation of the expression of pro-inflammatory markers CD86 and MHCII on macrophages, decrease the expression of the pro-inflammatory cytokine TNF- $\alpha$ and increase that of the anti-inflammatory cytokines IL-10 and TGF- $\beta 1(12,13)$. In addition, our earlier study has shown that the hypolipidemic effect of chitosan is partly attributed to its suppression of intestinal lipid absorption and hepatic acyl-coenzyme A: cholesterol acyltransferase-2 expression (18). Furthermore, chitosan has also been found to slow down the rate of tumor growth without inhibiting tumor formation (14); however, no detailed analysis of the immune responses in chitosan-treated animals, including mice, has been reported.

In the present study, normal BALB/c mice were randomly divided into four groups. The negative control group received a normal diet, the positive control group received a normal diet and acetic acid, and two treatment groups received a normal diet and the oral administration of 5 or $20 \mathrm{mg} / \mathrm{kg}$ chitosan 
in acetic acid. During the treatment, chitosan and/or acetic acid was administered, and the animals were weighed, every 2 days. At the end of the treatment period, blood samples were collected from all mice for cell marker analysis and measurement of phagocytosis, and splenocytes were isolated in order to examining NK cell activities and T- and B-cell proliferation.

To the best of our knowledge, this is the first study evaluating the effect of chitosan on immune responses in normal mice in vivo. The present results showed the following: i) chitosan did not significantly affect the appearance (Fig. 1A) or body (Fig. 1B), liver (Fig. 1C) and spleen (Fig. 1D) weights of the mice when compared with the acetic acid group; ii) chitosan increased CD3 (T cell; Fig. 2A), CD19 (B cell; Fig. 2B), CD11b (monocyte; Fig. 2C) and Mac-3 (macrophage; Fig. 2D) markers when compared with the acetic acid group; iii) chitosan treatment did not significantly increase the phagocytic activity of macrophages in PBMCs (Fig. 3A) but significantly decreased it in the peritoneal cavity (Fig. 3B); iv) chitosan at $20 \mathrm{mg} / \mathrm{kg}$ significantly decreased the cytotoxic effect of NK cells compared with that in the acetic acid group (Fig. 4); v) $20 \mathrm{mg} / \mathrm{kg}$ chitosan treatment significantly decreased $\mathrm{T}$ cell proliferation (Fig. 5A) compared with that in the acetic acid group, but B cell proliferation was not significantly affected by treatment with 5 and $20 \mathrm{mg} / \mathrm{kg}$ doses (Fig. 5B), and vi) chitosan decreased GOT and GPT activity compared with that in the acetic acid group, with GPT activity in the $20 \mathrm{mg} / \mathrm{kg}$ group being slightly higher than the levels in normal mice (Fig. 6B). Chitosan significantly increased LDH levels when compared with those in the acetic acid-treated group (Fig. 6C).

Chitosan promoted the cell markers CD3 (T cell), CD19 (B cell), CD11b (monocytes) and Mac-3 (macrophages) when compared with the acetic acid-treated mice. A previous study has reported that these four cell types play an important role in immune responses, particularly against the invasion of foreign antigens (19). Other studies have shown that macrophages play an important role in innate immunity $(20,21)$. Despite reports suggesting the involvement of chitosan in inflammatory responses, reliable data in the literature regarding the effects of chitosan on immune responses in normal mice in vivo are lacking. The aim of the present study, therefore, was to investigate the effects of chitosan on the immune responses of normal BALB/c mice in vivo.

A notable observation of the present study is that chitosan did not significantly affect the phagocytic activity of macrophages in PBMCs (Fig. 3A), but significantly decreased this activity in the peritoneal cavity (Fig. 3B); thus, the effects of chitosan on the Mac-3 marker and macrophage function require further study. It has been suggested that chitosan may exert an anti-inflammatory activity in astrocytoma cells (11) and macrophages $(12,13)$. Furthermore, it has been reported that chitosan exerts anti-inflammatory activity by modulating prostaglandin E synthase 2 levels through the c-Jun N-terminal kinase pathway, which has been suggested to be useful in the prevention or treatment of periodontal inflammation (22). Treatment with $20 \mathrm{mg} / \mathrm{kg}$ chitosan significantly decreased the cytotoxic effect of NK cells from normal mice. Compared with the acetic acid-treated group, chitosan did not significantly affect B-cell proliferation following LPS stimulation (Fig. 5B) but both doses of chitosan decreased T-cell proliferation following Con A stimulation (Fig. 5A). Further investigations are necessary to investigate this. It has been suggested that the great variability observed in chitosan samples, such as degrees of deacetylation, molecular weight, viscosity, and $\mathrm{pKa}$ may affect its properties (4).

Chitosan decreased the levels of GOT and GPT compared with those in the acetic acid-treated group; although the GPT level in the $20 \mathrm{mg} / \mathrm{kg}$ group was slightly higher than the level in normal mice (Fig. 6B). High levels of GPT and GOT activity in the serum have been recognized to be a reflection of hepatic cell destruction (23). The results of the present study indicate that chitosan may have a protective effect against hepatic cell death following exposure to acetic acid. Chitosan significantly increased LDH levels when compared with those in the acetic acid-treated group. Abnormal hepatic transaminase and LDH levels have been suggested to be associated with liver injury in patients with abdominal trauma (24). Acetic acid treatment in mice may lead to liver injury; on the basis of the increased levels of LDH in the blood observed in the present study following treatment with acetic acid and chitosan, it appears that chitosan may have a protective effect.

In conclusion, these findings suggest that chitosan modulates immune responses by increasing T-cell, B-cell, monocyte and macrophage cell markers in normal mice in vivo. Furthermore, comparisons between mice treated with acetic acid and chitosan, or chitosan alone indicate that chitosan treatment may protect against liver injury in vivo.

\section{Acknowledgements}

This study was supported by from China Medical University (Taichung, Taiwan; grant no. CMU102-ASIA-20) and Cheng Hsin General Hospital (Taipei, Taiwan; grant no. 103-01).

\section{References}

1. Jones LM, Broz ML, Ranger JJ, Ozcelik J, Ahn R, Zuo D, Ursini-Siegel J, Hallett M, Krummel M and Muller WJ: Stat3 establishes an immunosuppressive microenvironment during the early stages of breast carcinogenesis to promote tumor growth and metastasis. Cancer Res: 30 Dec, 2015 (Epub ahead of print).

2. Vinay DS, Ryan EP, Pawelec G, Talib WH, Stagg J, Elkord E, Lichtor T, Decker WK, Whelan RL, Kumara HM, et al: Immune evasion in cancer: Mechanistic basis and therapeutic strategies. Semin Cancer Biol (Suppl 35): S185-S198, 2015.

3. Vesely MD, Kershaw MH, Schreiber RD and Smyth MJ: Natural innate and adaptive immunity to cancer. Annu Rev Immunol 29: 235-271, 2011.

4. Domard A: A perspective on 30 years research on chitin and chitosan. Carbohydr Polym 84: 696-703, 2011.

5. Chung MJ, Park JK and Park YI: Anti-inflammatory effects of low-molecular weight chitosan oligosaccharides in IgE-antigen complex-stimulated RBL-2H3 cells and asthma model mice. Int Immunopharmacol 12: 453-459, 2012.

6. Francesko A and Tzanov T: Chitin, chitosan and derivatives for wound healing and tissue engineering. Adv Biochem Eng Biotechnol 125: 1-27, 2011

7. Ikinci G, Senel S, Akincibay H, Kaş S, Erciş S, Wilson CG and Hincal AA: Effect of chitosan on a periodontal pathogen Porphyromonas gingivalis. Int J Pharm 235: 121-127, 2002.

8. Choi BK, Kim KY, Yoo YJ, Oh SJ, Choi JH and Kim CY: In vitro antimicrobial activity of a chitooligosaccharide mixture against Actinobacillus actinomycetemcomitans and Streptococcus mutans. Int J Antimicrob Agents 18: 553-557, 2001.

9. Sarasam AR, Brown P, Khajotia SS, Dmytryk JJ and Madihally SV: Antibacterial activity of chitosan-based matrices on oral pathogens. J Mater Sci Mater Med 19: 1083-1090, 2008. 
10. Chung YC, Wang HL, Chen YM and Li SL: Effect of abiotic factors on the antibacterial activity of chitosan against waterborne pathogens. Bioresour Technol 88: 179-184, 2003.

11. Kim MS, Sung MJ, Seo SB, Yoo SJ, Lim WK and Kim HM: Water-soluble chitosan inhibits the production of pro-inflammatory cytokine in human astrocytoma cells activated by amyloid beta peptide and interleukin-1beta. Neurosci Lett 321: 105-109, 2002

12. Chou TC, Fu E and Shen EC: Chitosan inhibits prostaglandin E2 formation and cyclooxygenase-2 induction in lipopolysaccharide-treated RAW 264.7 macrophages. Biochem Biophys Res Commun 308: 403-407, 2003.

13. Yoon HJ, Moon ME, Park HS, Im SY and Kim YH: Chitosan oligosaccharide (COS) inhibits LPS-induced inflammatory effects in RAW 264.7 macrophage cells. Biochem Biophys Res Commun 358: 954-959, 2007.

14. Yeh MY, Wu MF, Shang HS, Chang JB, Shih YL, Chen YL, Hung HF, Lu HF, Yeh C, Wood WG, et al: Effects of chitosan on xenograft models of melanoma in C57BL/6 mice and hepatoma formation in SCID mice. Anticancer Res 33: 4867-4873, 2013.

15. Lu HF, Tung WL, Yang JS, Huang FM, Lee CS, Huang YP, Liao WY, Chen YL and Chung JG: In vitro suppression of growth of murine WEHI-3 leukemia cells and in vivo promotion of phagocytosis in a leukemia mice model by indole-3-carbinol. J Agric Food Chem 60: 7634-7643, 2012.

16. Nagamatsu Y, Yamamoto J, Fukuda A, Ohta M, Tsuda Y and Okada Y: Determination of leukocyte elastase concentration in plasma and serum by a simple method using a specific synthetic substrate. Haemostasis 21: 338-345, 1991.
17. No authors listed: Recommendations of the German Society for Clinical Chemistry. Standardization of methods for the determination of enzyme activities in biological fluids. Z Klin Chem Klin Biochem 8: 658-660, 1970.

18. Wu CC, Lin SY, Chen CT, Chang YP, Huang YS, Lii CK, Yu CC, Hsieh SL and Chung JG: Differential blood lipid-lowering effects of alkylsulfonated chitosan of different molecular weights in Syrian hamsters in vivo. Mol Med Rep 5: 688-694, 2012.

19. Arpinati M and Curti A: Immunotherapy in acute myeloid leukemia. Immunotherapy 6: 95-106, 2014.

20. Gordon S, Plüddemann A and Mukhopadhyay S: Sinusoidal immunity: Macrophages at the lymphohematopoietic interface. Cold Spring Harb Perspect Biol 7: a016378, 2015

21. Kim KH, Kim TS, Lee JG, Park JK, Yang M, Kim JM, Jo EK and Yuk JM: Characterization of proinflammatory responses and innate signaling activation in macrophages infected with Mycobacterium scrofulaceum. Immune Netw 14: 307-320, 2014.

22. Arancibia R, Maturana C, Silva D, Tobar N, Tapia C, Salazar JC, Martínez J and Smith PC: Effects of chitosan particles in periodontal pathogens and gingival fibroblasts. J Dent Res 92: 740-745, 2013.

23. Yamashita T, Ohshima H, Asanuma T, Inukai N, Miyoshi I, Kasai N, Kon Y, Watanabe T, Sato F and Kuwabara M: The effects of alpha-phenyl-tert-butyl nitrone (PBN) on copper-induced rat fulminant hepatitis with jaundice. Free Radic Biol Med 21: 755-761, 1996.

24. Bilgic I, Gelecek S, Akgun AE and Ozmen MM: Predictive value of liver transaminases levels in abdominal trauma. Am J Emerg Med 32: 705-708, 2014. 